\title{
Generating femtosecond X-ray pulses using an emittance-spoiling foil in free-electron lasers
}

\author{
Y. Ding ${ }^{1}$, C. Behrens ${ }^{2}$, R. Coffee ${ }^{1}$, F.-J. Decker ${ }^{1}$, P. Emma ${ }^{1}$, C. Field ${ }^{1}$, W. Helml ${ }^{3}$, Z. Huang ${ }^{1}$, \\ P. Krejcik ${ }^{1}$, J. Krzywinski ${ }^{1}$, H. $\operatorname{Loos}^{1}$, A. Lutman ${ }^{1}$, A. Marinelli ${ }^{1}$, T. J. Maxwell ${ }^{1}$, J. Turner ${ }^{1}$ \\ ${ }^{1}$ SLAC National Accelerator Laboratory, Menlo Park, California 94025, USA \\ 2 Deutsches Elektronen-Synchrotron DESY, Notkestr. 85, 22607 Hamburg, Germany \\ 3 Technische Universität München, James-Franck-Stra $\beta$ e 1, 85748 Garching, Germany
}

\begin{abstract}
Generation of femtosecond to sub-femtosecond pulses is attracting much attention in X-ray freeelectron laser user community. One method is to use a slotted, emittance-spoiling foil which was proposed before (P. Emma, K. Bane, M. Cornacchia, Z. Huang, H. Schlarb, G. Stupakov, and D. Walz, Phys. Rev. Lett., 92, 074801, 2004.) and has been widely used at the Linac Coherent Light Source. Direct experimental characterization of slotted-foil performance was previously unfeasible due to a lack of appropriate diagnostics. With a recently installed X-band radio-frequency transverse deflector, we are able to characterize the electron bunch spoiling effect and X-ray pulse when using the slotted foil. We show that few-femtosecond X-ray pulses are generated with flexible control of the single-pulse duration or double-pulse separation with comparison to the theoretical model.
\end{abstract}

X-ray free-electron lasers (FELs) provide a unique tool for ultrashort time-resolved X-ray studies in the femtosecond regime. This enables different dynamic process studies such as measuring the molecular motions in the ephemeral transition states of physical, chemical and biological systems [1]. During these studies, femtosecond time resolution and flexible control of the pulse duration are critical to resolve the dynamics of the chemical bond at the atomic level.

At present X-ray FEL facilities such as the Linac Coherent Light Source (LCLS) [2], the typical pulse duration is about a few 10s to 100s of femtoseconds with a regular operating charge of $150-250 \mathrm{pC}$. To achieve even shorter pulses, a lower charge $(20 \mathrm{pC})$ has been used for producing less than 10 -fs X-rays [3, 4]. Switching to this low-charge mode typically requires additional accelerator tuning and X-ray focusing optics adjustments. Another way to generate shorter FEL pulses is using an emittance-spoiling foil [5], which has been in use at the LCLS since 2010. In this scheme, an aluminum foil with slots is installed at a dispersive section of the accelerator beamline. After electrons scatter from the foil, most of the beam will not lase due to increased emittance, while the small, unspoiled fraction which passes through the slot will contribute to lasing. The pulse duration is simply controlled by choosing different slot sizes. It can also deliver a pair of closely spaced X-ray pulses by using a double-slotted foil. The ease of operation and flexibility of pulse control made the slotted foil mode popular in some user applications [6].

Although the slotted-foil mode has been widely used during LCLS operation, due to the absence of appropriate femtosecond-scale diagnostics, the absolute FEL pulse duration or double-pulse separation has only been estimated from formulas in past years. In fact, for a very narrow slot, the pulse duration is partially affected by energy spread and emittance which makes this estimation less accurate. In addition, the pulse duration or separation fluctuates due to electron beam energy jitter. In many experiments such as nonlinear X-ray sciences and molecular imaging studies, precise knowledge of the pulse duration on a shot-by-shot basis is essential for interpreting the data.

Experimental characterization of femtosecond X-rays is very challenging and different methods have been explored in recent years [7]. For example, direct characterization of the X-ray pulses by streaking spectroscopy [8$10]$ has been demonstrated, but requires a specific experimental set-up not yet readily available as a diagnostic for user experiments. Recently, at the LCLS, another method by measuring the lasing "footprint" on the electron beam with an X-band radio-frequency (RF) transverse deflecting cavity (XTCAV) $[4,11]$ has been developed as an online electron and X-ray temporal diagnostic. This provides a single-shot diagnostic at the full beam repetition rate. Since it actually measures the electron beam longitudinal phase space, we can also use it to characterize the emittance-spoiling effect.

In this Letter we report experimental studies of generating and controlling femtosecond X-ray FEL pulses using an emittance-spoiling foil. We present direct singleshot diagnosis using the XTCAV and compare measured data with theoretical calculations. In this way we verify the full achievable operational range as additional guidance for, in general, the accelerator-based light source community and, in particular, potential ultrafast X-ray applications at the LCLS.

Figure 1 shows a schematic of the LCLS layout from the second linac section (L2) to the beam dump. The L2 linac is set at an off-crest accelerating rf phase, so that the beam energy after L2 will be correlated with time. When the beam travels to the middle of the second bunch compressor (BC2), the time-energy correlated beam will be tilted at a large angle relative to the beam propagation axis. A 3- $\mu \mathrm{m}$ thickness aluminum foil with vertically varying size slot is placed in the path of the beam at the middle of $\mathrm{BC} 2$. Coulomb scattering increases the emittance of most of the beam which passes through the foil, 


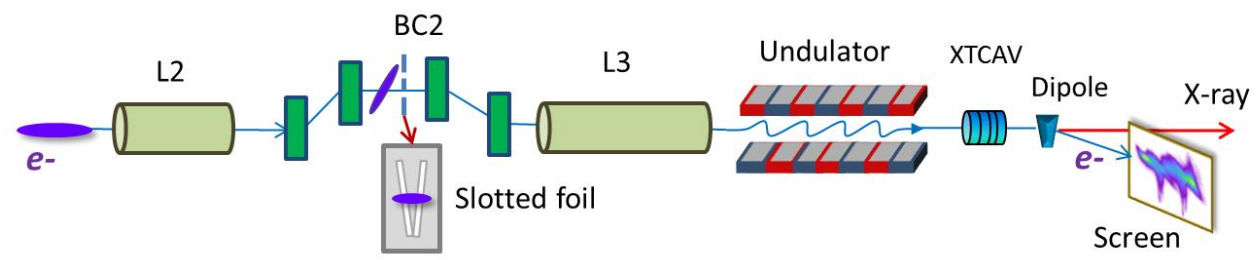

FIG. 1. A schematic of the LCLS machine layout including an emittance spoiling foil in the middle of second bunch compressor (BC2) and the XTCAV downstream of the FEL undulator. The L2 and L3 are S-band RF linac sections. A double-slotted foil is used here for an illustration.

while a very thin unspoiled slice passing through the slot still has its emittance preserved [5]. In this configuration, only the unspoiled slice contributes to lasing. For a flexible control of the output pulses, the designed foil has different available slot geometries [12], the popular ones including a variable-width single slot and two V-shape double slots, each with a different, fixed slot width.

For a time-energy chirped beam we define a linear chirp strength $h$ by $\delta=h z_{0}$, where $\delta$ and $z_{0}$ are the electrons' relative energy spread and longitudinal coordinate before compression, respectively. After the bunch compressor with a momentum compaction factor $R_{56}$ [13] and a momentum dispersion $\eta$ at the middle of the chicane, the centroid time separation $\Delta t$ of the two pulses generated from a double-slot geometry can be written as

$$
\Delta t=\frac{\Delta d}{|\eta h| C_{b} c}
$$

where $\Delta d$ is the centroid distance between the double slots, $C_{b}=1 /\left(1+h R_{56}\right)$ is the bunch compression factor for a cold beam, and $c$ is the speed of light in vacuum. Similarly, we can also calculate the pulse duration $\Delta \tau$ from a slot with a finite width, but the uncorrelated energy spread and transverse betatron beam size have to be included, as discussed in [14]:

$$
\Delta \tau \approx \frac{2.35}{|\eta h| c} \sqrt{\eta^{2} \sigma_{\delta_{0}}^{2}+\left(\Delta x^{2} / 12+\sigma_{x_{\beta}}^{2}\right) / C_{b}^{2}} .
$$

Here $\sigma_{\delta_{0}}$ is the initial uncorrelated relative energy spread before compression, $\Delta x$ is the slot full width, and $\sigma_{x_{\beta}}$ is the rms betatron beam size at the foil. Comparing to Eq. (1), we can see the beam uncorrelated energy spread and betatron size also affect the pulse duration.

In the undulator, the FEL lasing from the unspoiled slice will induce time-dependent energy spread growth and energy loss. This lasing "footprint" on the electron longitudinal phase space is measured directly with the XTCAV diagnostic [4]. The XTCAV provides a timedependent horizontal deflection to the electron bunch, followed by a DC, vertically bending spectrometer magnet. With this setup, we make absolute measurements of the beam time and energy distribution with knowledge of the deflecting cavity strength and the bend angle.

Figure 2 shows two measured examples for electrons passing through the single slot with different slot widths.
The electron bunch is operated in under-compression, with beam parameters shown in Table I. In subplots (b) - (d), the slot full-width is $760 \mu \mathrm{m}$. We can see directly in subplot (b) that the unspoiled fraction at the core part has a much smaller energy spread than the rest of the bunch when lasing is suppressed. Although the foil spoils the emittance rather than the energy spread, the increased beam divergence due to Coulomb scattering couples to the longitudinal dimensions through the last half of the chicane, hence the uncorrelated energy spread is increased. The FEL lasing of the unspoiled portion generates much larger slice energy spread as shown in subplot (c). Comparing (b) with (c), the X-ray profile is reconstructed in subplot (d). The detailed reconstruction method can be found in [4]. In this example the generated X-ray length is $25 \mathrm{fs}$ fwhm.

With a narrower slot (full width $\sim 270 \mu \mathrm{m}$ ), we see a much smaller unspoiled fraction (Fig. 2 (e) and (f)) and the resultant X-ray pulse is about $10 \mathrm{fs}$ fwhm. Also note a spike in the current profile is directly observed, as predicted in [5]: scattered electrons travel with a slightly different path length through the last half of the chicane and overlap in time with the unspoiled time slice. This timesmearing of spoiled electrons leads to the prominence of the current spike. Although the FEL only amplifies the cold beam core and is not affected by the time-smeared halo, this current spike still causes additional electron energy modulation from the downstream longitudinal space charge and wake fields. This leads to the observed tilt (chirp) in the unspoiled beam portion, especially for the narrow slot case as shown in subplot (e).

With a double-slot geometry, we can generate two pulses for X-ray pump-probe experiments. The LCLS double slot has a $\mathrm{V}$ shape (with centroid separation range 0.6-1.4 $\mathrm{mm}$ ) to control the pulse separation, with either thin slots (slot full width $\sim 300 \mu \mathrm{m}$ ) or thicker slots (slot full width $\sim 430 \mu \mathrm{m}$ ) (see subplot (a) in Fig. 3). We show two measured examples in Fig. 3 with the beam at the middle of each double slot (the slot centroid separation is equal). The measured pulse separation is about the same as expected, but clearly the individual pulse duration in each pair is different. The stability between the double pulses are essential for some pump-probe experiments. In this example, based on 25 shots measurement, the measured temporal separation jitter between 

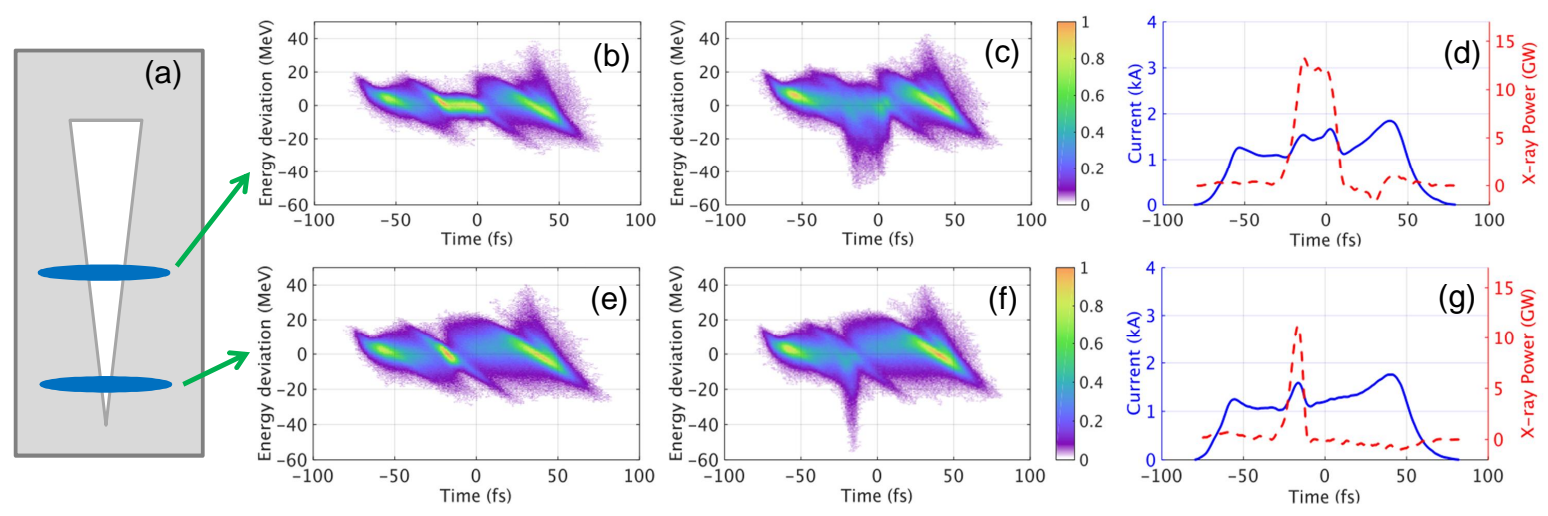

FIG. 2. Measurement examples for the single-slot foil. At two different slot widths (a), the longitudinal phase spaces with lasing-off $((\mathrm{b})$ and $(\mathrm{e}))$ and lasing-on $((\mathrm{c})$ and $(\mathrm{f}))$ are measured. The current profile and reconstructed X-ray power profile are shown in (d) and (g) for each slot position. The measured X-ray pulse durations are 25 fs and $10 \mathrm{fs}$ fwhm, respectively. Beam parameters are listed in Table. I.

the double pulses is $9 \%$, and the relative peak power jitter $\left(\left(P_{1}-P_{2}\right) /\left(P_{1}+P_{2}\right), P_{1,2}\right.$ is the peak power of the two pulses in a pair) is about $10 \%$. These fluctuations can be measured shot by shot at the full beam repetition rate from XTCAV so the experimental data can be sorted accordingly.

Similar to the examples shown in Figs. 2 and 3, we systematically measured the pulse duration and separation at different single-slot widths and double-slot separations. Each point was averaged from 25 single-shot measurements. The main beam parameters are listed in Table I, which are also used for calculations with Eqs. (1) and (2). Measurement and calculation results are plotted in Fig. 4. We see the pulse duration or separation are well controlled purely by adjusting the foil's vertical position, and the calculated results agree reasonably well with the measured data. At the larger double-slot separations, the measured pulse separation is slightly smaller than that from calculation. This is due to a non-uniform distribution of the beam current: the beam current near the bunch head and tail is actually higher with a doublehorn current profile, while the calculation assumes a uniform current profile which under-estimates the compression factor $C_{b}$ and the chirp $h$ near the horns. This $\sim 1 \mathrm{kA}$ current level is typical for soft X-ray $(<2 \mathrm{keV})$ operation at the LCLS. For hard X-ray FELs, the beam current is typically $3-4 \mathrm{kA}$ with a larger chirp and compression, so that the generated pulse duration and separation are smaller. Calculated achievable pulse duration and separation at different currents can be found in [15].

Low-charge $(20 \mathrm{pC})$ operation at the LCLS can produce less than $10 \mathrm{fs} \mathrm{X}$-rays $[3,4]$. In this mode the bunch is strongly compressed in $\mathrm{BC} 2$ to reach kA-level peak currents. Since the chirp $h$ and compression factor $C_{b}$ are larger, it could achieve even shorter pulses if we combine the slotted-foil scheme with the 20-pC mode [16]. With $20-\mathrm{pC}$ charge, we operate in over-compression which produces a Gaussian-like current profile but with a residual
TABLE I. Main parameters for slotted-foil measurements.

\begin{tabular}{lccc}
\hline \hline Parameter & Symbol & Value & Unit \\
\hline Bunch charge & $\mathrm{Q}$ & 180 & $\mathrm{pC}$ \\
Beam energy at BC2 & $E$ & 5 & $\mathrm{GeV}$ \\
BC1 current & $I_{1}$ & 220 & $\mathrm{~A}$ \\
BC2 current & $I_{2}$ & 1100 & $\mathrm{~A}$ \\
Uncorrelated E-spread before BC2 & $\sigma_{\delta_{0}}$ & 130 & $\mathrm{keV}$ \\
energy chirp before BC2 & $h$ & 38 & $\mathrm{~m}^{-1}$ \\
Betatron beam size rms & $\sigma_{x_{\beta}}$ & 38 & $\mu \mathrm{m}$ \\
Dispersion at foil location & $\eta$ & 0.36 & $\mathrm{~m}$ \\
Momentum compaction & $R_{56}$ & -24.7 & $\mathrm{~mm}$ \\
Single-slot full-width & $\Delta x$ & $0.27-1.44$ & $\mathrm{~mm}$ \\
Double-slot centroid separation & $\Delta d$ & $0.64-1.42$ & $\mathrm{~mm}$ \\
FEL wavelength & $\lambda$ & 1.1 & $\mathrm{~nm}$ \\
\hline \hline
\end{tabular}

time-energy chirp. The slotted foil selects the central high-current core for lasing.

We show two measured examples in Fig. 5 for a bunch charge of $20 \mathrm{pC}$ and a beam energy of $4.8 \mathrm{GeV}$. At the middle of the slot (subplot (b) - (d)), the measured pulse duration is $9 \mathrm{fs}$ fwhm, and at the bottom (subplot (e) (f)) it is $3.8 \mathrm{fs}$ fwhm. The XTCAV resolution is $\sim 1 \mathrm{fs}$ rms at this energy [4] which is a small effect for regular pulses whose durations are much longer. But for this short pulse of $3.8 \mathrm{fs}$ fwhm, the actual pulse duration could be $20 \%$ shorter after subtracting the resolution. On the other hand, the FEL slippage after saturation may lengthen the X-ray pulse duration which is not directly measurable on the electron beam (i.e., with the $\mathrm{XTCAV}$ ). For example, the slippage length is about $1 \mathrm{fs}$ with $10 \mathrm{~m}$ of post-saturation undulator at this energy. To generate short FEL pulses, we therefore generally avoid operating in the deep saturation regime to mitigate the slippage effect. The influence of resolution and slippage are negligible for long pulses, but for very short pulse op- 

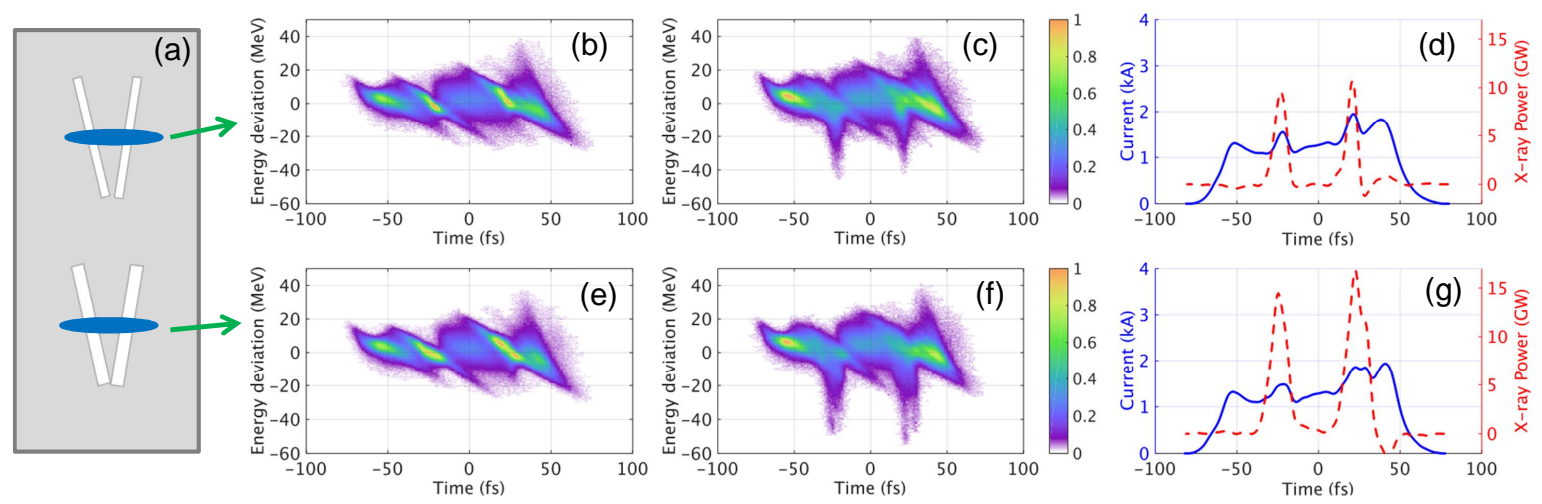

FIG. 3. Measurement examples for the double-slotted foil. The examples are measured at the middle of the thin and thick double-slotted geometries. The lasing-off images are shown in ((b) and (e)), and the lasing-on images in ((c) and (f)). The current profile and reconstructed X-ray profile are shown in $(\mathrm{d})$ and $(\mathrm{g})$. The double-pulse separation $(\sim 45 \mathrm{fs})$ is the same for the two sets but the pulse duration is different. Beam parameters are listed in Table I.

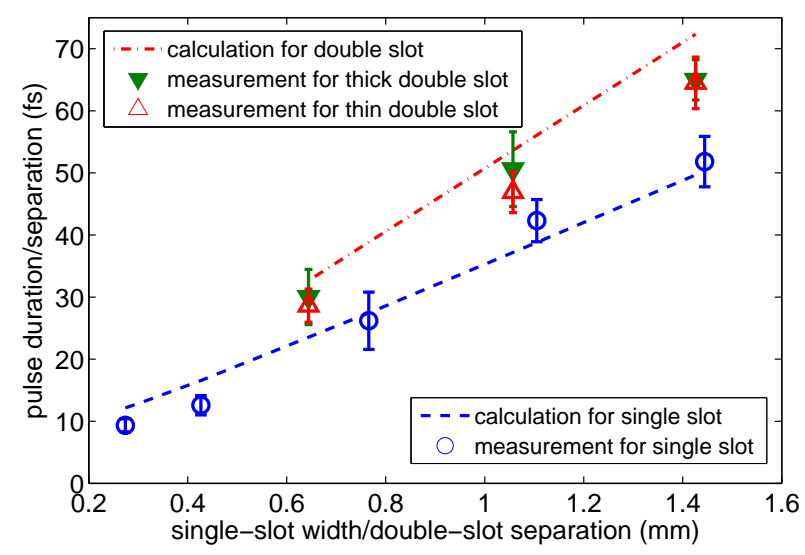

FIG. 4. Experimental measurements and calculations of the pulse duration versus single-slot width (dashed line and circles) and double-pulse separation versus double-slot separation (dashed dotted line and triangles). Each data point is averaged with 25 single-shot measurements. Beam parameters are listed in Table I.

eration other X-ray diagnostic methods would be helpful for a benchmark once available. Also we note that with a time-energy chirped beam as when in over-compression, we can generate two-color double pulses when using the double slot. Compared to other two-color FEL operation modes [17-19], the color separation is correlated with temporal separation in this configuration which causes less flexibility for independent time/energy adjustment.

For hard X-ray energies, as discussed earlier, the achievable pulse duration and separation can be even smaller at the required higher operating currents. On the other hand, the XTCAV resolution is lower (the measured XTCAV resolution is $\sim 4 \mathrm{fs}$ rms at $9 \mathrm{keV}$ [4]). For an extended characterization of the slotted foil with hard $\mathrm{X}$-rays, a spectral domain method at the LCLS is under study [20].

In summary, we have experimentally demonstrated a flexible control of the pulse duration or separation in Xray FELs with an emittance-spoiling foil. The measured data show that short pulses down to a few femtoseconds can be generated in the soft X-ray regime. The pulse duration or double-pulse separation is adjustable by choosing the slot size, and the measurement agrees reasonably well with the theoretical model. In hard X-ray regime, shorter pulses are expected and spectral domain diagnostics is planned for a full characterization.

This work was supported by Department of Energy Contract No. DE-AC02-76SF00515. We thank SLAC operations and engineering groups for their dedicated support. We also thank LCLS user groups for their feedback on using the slotted foil mode.
[1] A. H. Zewail, J. Phys. Chem. A 104, 5660 (2000).

[2] P. Emma, R. Akre, J. Arthur, R. Bionta, C. Bostedt, J. Bozek, A. Brachmann, P. Bucksbaum, R. Coffee, F.-J. Decker et al., Nat. Photon. 4, 641 (2010).

[3] Y. Ding, A. Brachmann, F.-J. Decker, D. Dowell, P. Emma, J. Frisch, S. Gilevich, G. Hays, Ph. Hering, Z. Huang et al., Phys. Rev. Lett, 102, 254801 (2009).

[4] C. Behrens, F.-J. Decker, Y. Ding, V.A. Dolgashev, J.
Frisch, Z. Huang, P. Krejcik, H. Loos, A. Lutman, T.J. Maxwell et al., Nat. Communications 5, 3762 (2014).

[5] P. Emma, K. Bane, M. Cornacchia, Z. Huang, H. Schlarb, G. Stupakov, and D. Walz, Phys. Rev. Lett., 92, 074801 (2004).

[6] For example, see a review paper, C Bostedt, J. D. Bozek, P. H. Bucksbaum, R. N. Coffee, J. B. Hastings, Z. Huang, R. W. Lee, S. Schorb, J. N. Corlett, P. Denes et al., J. 

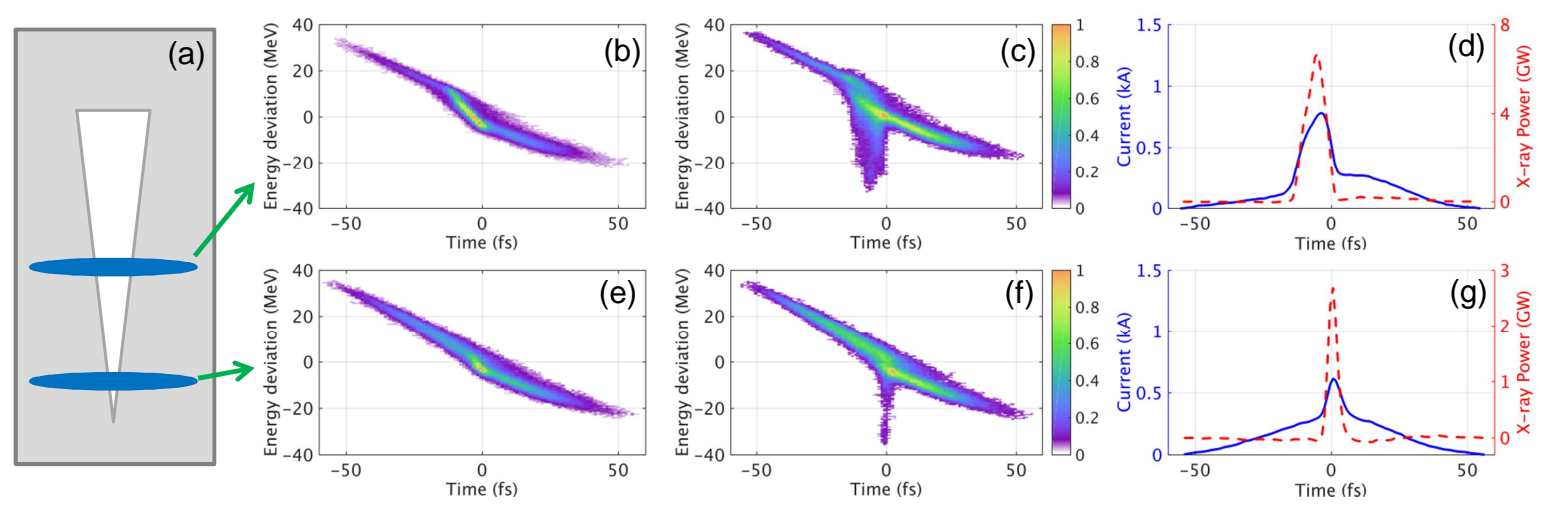

FIG. 5. Measurement examples at two slot widths for a $20 \mathrm{pC}, 4.8 \mathrm{GeV}$ beam, operated in an over-compression mode. The lasing-off images are shown in ((b) and (e)), and the lasing-on images in ((c) and (f)). The current profile and reconstructed X-ray profile are shown in $(\mathrm{d})$ and $(\mathrm{g})$. The X-ray pulse duration in these two examples are 9 and 3.8 fs, respectively.

Phys. B: At. Mol. Opt. Phys. 46164003 (2013).

[7] For example, see a review paper, S. Düsterer, Phys. Rev. ST Accel. Beams 17, 120702 (2014).

[8] Ulrike Fr'uhling, Marek Wieland, Michael Gensch, Thomas Gebert, Bernd Sch'utte, Maria Krikunova, Roland Kalms, Filip Budzyn, Oliver Grimm, J'org Rossbach et al. Nat. Photon. 3, 523-528 (2009).

[9] I. Grguraš, A. R. Maier, C. Behrens, T. Mazza, T. J. Kelly, P. Radcliffe, S. D'usterer, A. K. Kazansky, N. M. Kabachnik, Th. Tschentscher et al. Nat. Photon. 6, 852857 (2012).

[10] W. Helml, A. R. Maier, W. Schweinberger, I. Grguraš, P. Radcliffe, G. Doumy, C. Roedig, J. Gagnon, M. Messerschmidt, S. Schorb et al., Nat. Phot. 8, 950 (2014)

[11] Y. Ding, C. Behrens, P. Emma, J. Frisch, Z. Huang, H. Loos, P. Krejcik, and M-H. Wang, Phys. Rev. ST Accel. Beams 14, 120701 (2011).

[12] Y. Ding, F.-J. Decker, P. Emma, C. Feng, C. Field, J. Frisch, Z. Huang, J. Krzywinski, H. Loos, J. Welch et al., Phys. Rev. Lett., 109, 254802 (2012).

[13] K. Brown and R. Servrancks, SLAC-PUB-3381 (1984).

[14] P. Emma, Z. Huang, and M. Borland, Proceedings of FEL 2004, Trieste, Italy.

[15] Y. Ding, A. Brachmann, F.-J. Decker, P. Emma, C. Field, J. Frisch, Z. Huang, R. Iverson, H. Loos, H.-D. Nuhn et al., Proceedings of IPAC13, Shanghai, China, 2013.

[16] L. Wang, Y. Ding and Z. Huang, Proceedings of IPAC11, San sebastian, Spain, 2011.

[17] A. A. Lutman, R. Coffee, Y. Ding, Z. Huang, J. Krzywinski, T. Maxwell, M. Messerschmidt, and H.-D. Nuhn, Phys. Rev. Lett., 110, 134801 (2013).

[18] A. Marinelli, A. A. Lutman, J. Wu, Y. Ding, J. Krzywinski, H.-D. Nuhn, Y. Feng, R. N. Coffee, and C. Pellegrini, Phys. Rev. Lett., 111, 134801 (2013).

[19] A. Marinelli, D. Ratner, A.A. Lutman, J. Turner, J. Welch, F.-J. Decker, H. Loos, C. Behrens, S. Gilevich, A.A. Miahnahri et al., Nat. Communications 6, 6369 (2015).

[20] T. J. Maxwell and D. Zhu, SLAC National Accelerator Laboratory, private communication. 